\title{
Negatively Charged Oxygen Ions as a Plasma Diagnostic Tool: Application to Comets
}

\author{
Sreekala Gopinathan', Sijo Sebastian1, Noble Abraham¹, Savithri Devi', \\ Venugopal Chandu ${ }^{*}$, Renuka Gangadharan ${ }^{2}$ \\ ${ }^{1}$ School of Pure \& Applied Physics, Mahatma Gandhi University, Kottayam, India \\ ${ }^{2}$ Kerala State Council for Science, Technology \& Environment, Thiruvananthapuram, India \\ Email: ${ }^{*}$ cvgmgphys@yahoo.co.in
}

Received 3 May 2014; revised 13 June 2014; accepted 23 July 2014

Copyright (C) 2014 by authors and OALib.

This work is licensed under the Creative Commons Attribution International License (CC BY).

http://creativecommons.org/licenses/by/4.0/

(c) (i) Open Access

\begin{abstract}
We study the stability of the ion-acoustic (IA) wave in a collisional plasma composed of hydrogen, positively and negatively charged oxygen ions, electrons and neutral atoms. This composition approximates very well the plasma environment around a comet. A solution of the dispersion relation yields a frequency for the IA wave at around the hydrogen plasma frequency. The growth/ damping rate is sensitively dependent on the ring parameters $u_{\perp s}$ (the ring speed) and $v_{t s}$ (the thermal spread). The growth rate of the wave, which decreases with increasing collisional frequencies, is larger when $u_{\perp s}<v_{t s}$. In the presence of negatively charged oxygen ions, the phase and group velocities of the IA wave behave in a contrasting manner when $u_{\perp s}<v_{t s}$ (and viceversa). We propose that this behaviour be exploited as a diagnostic tool for the detection of these ions and also their thermalization.
\end{abstract}

Keywords

Ion-Acoustic Wave, Negative Ions, Diagnostic Tool, Cometary Plasma

Subject Areas: Plasma, Theoretical Physics

\section{Introduction}

One of the most fundamental of waves in a plasma is the low frequency electrostatic or longitudinal ion density wave; in the long-wavelength limit the ions provide the inertia and the electrons, the restoring force [1] [2]. The ion-acoustic (IA) waves are usually highly Landau damped unless $T_{i} \ll T_{e}$; where $T_{i}$ and $T_{e}$ are the ion and electron temperatures respectively [3] [4]. This wave has been invoked to explain a number of phenomena in space plasmas, such as the observed wave characteristics in Earth's ionosphere [5], transport in the solar wind, 
corona and chromosphere [6]. It has also been studied extensively in a number of laboratory plasmas [7] [8].

A cometary environment is believed to consist of hydrogen and new born heavier ions, such as positively charged oxygen ions with densities which depend on their distances from the nucleus [9]. However, the spacecraft Giotto, which observed the inner coma of comet Halley showed that, in addition to neutral atoms and molecules, thermal ions and electrons, etc. a new component, namely negatively charged ions was also present [10]. The negative ions were observed in the mass peaks of 7 - 19, 22 - 65 and 85 - 110 amu with negatively charged oxygen ions being identified unambiguously.

A cometary plasma has often been modelled as a solar wind plasma, permeated by dilute, drifting ring distribution of ions and electrons with finite thermal spreads. Instabilities driven by electron velocity ring distributions have been well studied. For example, for cyclotron harmonic wave propagation perpendicular to the static magnetic field, it was found that mode coupling as well as a strong absolute instability occurs [11]. In a pioneering experiment on the electron cyclotron maser oscillator, Sprangle et al. [12] analyzed a configuration that utilized an open-resonator cavity containing a gyrating electron beam, which translated along an external magnetic field directed transverse to the axis of symmetry of the resonator. Upon entering the resonator, the electrons were assumed to have the same transverse and parallel velocities [12]. Studies using these distributions have also been criticized as being too restrictive to describe particle distributions observed in certain regions of space plasmas; for example, the magnetosphere [13].

However, ion ring distributions are more important because of the greater amount of free energy available [14]. Instability studies driven by such distributions include the electrostatic ion cyclotron instability [15] [16], the lower-hybrid instability [17]-[20] and the ion-acoustic instability [14].

The IA wave is one that has been observed frequently in the plasma environments of comets: for example, this wave was observed in the frequency range of $1-1.5 \mathrm{kHz}$ at the comet Giacobini-Zinner [21] and around 1 $\mathrm{kHz}$ at comet Halley [22].

We have, therefore, studied the stability of the IA wave in a six component collisional plasma consisting of solar wind protons and electrons and cometary hydrogen, and positively and negatively charged oxygen ions and neutrals. We find that collisions with neutrals only reduce the growth rates lightly in spite of the high neutral densities. On the other hand, the phase and group velocities of the IA wave are sensitively dependent on the ratio of the ring speed $\left(u_{\perp s}\right)$ to the thermal spread $\left(v_{t s}\right)$ of the negatively charged oxygen ions $\left(\mathrm{O}^{-}\right)$. This variation in the phase and group velocities when $u_{\perp s}>v_{t s}$ (and vice-versa) is proposed as a tool to detect the presence of $\mathrm{O}^{-}$ions and also their thermalization.

\section{The Dispersion Formula}

As stated above we are interested, in this paper, on the propagation characteristics of the IA wave in a plasma composed of protons and electrons (of solar wind origin) and hydrogen and positively and negatively charged oxygen ions (of cometary origin); the neutral atoms and molecules form the sixth component. The protons and electrons of solar wind origin are modeled by drifting Maxwellian distributions while the ions of cometary origin are modelled by ring distributions. Combining the contributions of the various species, we can write the dispersion formula for IA waves of frequency $\omega$ and wave vector $\boldsymbol{k}$ in this multi-component plasma as [14] [23]

$$
D(\omega, \boldsymbol{k})=1+\sum_{j=p, e} \chi_{j}+\sum_{s=\mathrm{H}^{+}, \mathrm{O}^{+}, \mathrm{O}^{-}} \chi_{s}^{r}=0
$$

In (1)

$$
\begin{gathered}
\chi_{e}=\frac{1}{k^{2} \lambda_{D e}^{2}} \frac{\left[1+\zeta_{e} \Gamma_{0}\left(b_{e}\right) Z\left(\zeta_{e}\right)\right]}{\left[1+\frac{i v_{e}}{\sqrt{2} k_{z} v_{T e}} \Gamma_{0}\left(b_{e}\right) Z\left(\zeta_{e}\right)\right]} \\
\chi_{p}=\frac{1}{k^{2} \lambda_{D p}^{2}} \frac{\left[1+\zeta_{p} Z\left(\zeta_{p}\right)\right]}{\left[1+\frac{i v_{p}}{\sqrt{2} k_{z} v_{T p}} Z\left(\zeta_{p}\right)\right]}
\end{gathered}
$$


and

$$
\chi_{s}^{r}=-\left(\omega_{p s}^{r}\right)^{2} \frac{\omega}{\left[\omega^{2}-k_{\perp}^{2}\left(u_{\perp s}-i v_{t s}\right)^{2}\right]^{3 / 2}}
$$

(2a) and (2b) are the contributions of the electrons and protons of solar wind origin while (2c) are the contributions from the cometary ions; hydrogen $\left(\mathrm{H}^{+}\right)$, positively charged oxygen $\left(\mathrm{O}^{+}\right)$and negatively charged oxygen $\left(\mathrm{O}^{-}\right)$. In (2a) and (2b), $\lambda_{D j}$ is the Debye length of electrons (protons) while $k_{z}$ is the wave vector component parallel to the magnetic field. The argument of the plasma dispersion function is

$$
\zeta_{e(p)}=\frac{\omega-k_{z} u_{0 e(p)}+i v_{e(p)}}{\sqrt{2} k_{z} v_{T e(p)}}
$$

In (3) $u_{0 e(p)}$ is the drift velocity of the electrons (protons) while $v_{e(p)}$ is the collision frequency of the neutral atoms with electrons (protons) and $v_{T e(p)}$ is the thermal velocity of electrons (protons) $\left(v_{T e(p)}=\left[T_{e(p)} / m_{e(p)}\right]^{1 / 2}\right)$. Finally $\Gamma_{0}\left(b_{e}\right)$, which arises from the $d v_{\perp}$-integration, is defined by

$$
\Gamma_{0}\left(b_{e}\right)=\mathrm{e}^{-b_{e}} I_{0}\left(b_{e}\right)
$$

where $I_{0}\left(b_{e}\right)$ is the modified Bessel function of order zero with an argument

$$
b_{e}=\frac{k_{\perp}^{2} T_{e}}{\Omega_{e}^{2} m_{e}}
$$

$\Omega_{e}$ is the electron gyro-frequency while $k_{\perp}$ is the wave vector perpendicular to the magnetic field.

In (2c) $\omega_{p s}^{r}$ is the plasma frequency of the species $s=\mathrm{H}^{+}, \mathrm{O}^{+}, \mathrm{O}^{-}$while $u_{\perp s}$ are the beam velocities of the species $s$ perpendicular to the magnetic field and $v_{t s}$ are their thermal spreads.

\section{The Dispersion Relation}

In this section we derive the dispersion relation for IA waves in the plasma under consideration. We consider IA waves in the frequency regime

$$
v_{T P} \ll \frac{\omega}{k_{z}} \ll v_{T e}, \quad u_{0 e(p)} \ll v_{T e(p)}
$$

We thus need the small parameter expansion of the plasma dispersion function for electrons and its asymptotic expansion for the protons. They are given respectively [24] by

$$
\begin{aligned}
& Z\left(\zeta_{e}\right)=-2 \zeta_{e}+\frac{4}{3} \zeta_{e}^{3}+\cdots+i \sqrt{\pi} \mathrm{e}^{-\zeta_{e}^{2}}, \quad \zeta_{e} \ll 1 \\
& Z\left(\zeta_{p}\right)=-\frac{1}{\zeta_{p}}-\frac{1}{2} \frac{1}{\zeta_{p}^{3}}+\cdots+i \sqrt{\pi} \frac{k}{|k|} \mathrm{e}^{-\zeta_{p}^{2}}, \quad \zeta_{p} \gg 1
\end{aligned}
$$

We consider two contrasting cases namely that of small and large thermal spreads of the ring ions separately.

\subsection{Large Thermal Spreads for Ring Ions $\left(v_{t s}>u_{\perp s}\right)$}

Using the expansions (7a) in (2a) and (7b) in (2b) for the solar wind electrons and protons and the approximation $v_{t s}>u_{\perp s}$ in (2c) for the ring ions, the contributions $\chi_{e}, \chi_{p}$ and $\chi_{s}^{r}$ to the dispersion formula (1) can be easily calculated. Using these, the expression for the real frequency and the growth/damping rate $\left(=-\operatorname{ImD} /\left(\partial \operatorname{ReD} / \partial \omega_{r}\right)\right)$ can be derived as

$$
\omega_{r}^{2}=\omega_{p p}^{2} \frac{k^{2} \lambda_{D e}^{2}}{\left(1+k^{2} \lambda_{D e}^{2}\right)} A
$$

and 


$$
\begin{aligned}
\gamma= & -\sqrt{\frac{\pi}{8}} \frac{\omega_{r}}{\left(1+k^{2} \lambda_{D e}^{2}\right)}\left[\frac{\omega_{r}-k_{z} u_{0 e}}{k_{z} v_{T e}} \Gamma_{0}\left(b_{e}\right) \mathrm{e}^{-\zeta_{e, r}^{2}} \times\left(1+\sqrt{\frac{\pi}{2}} \frac{v_{e}}{k_{z} v_{T e}} \Gamma_{0}\left(b_{e}\right) \mathrm{e}^{-\zeta_{e, r}^{2}}\right) \frac{A}{B}\right] \\
& -\frac{\omega_{r}}{2} \frac{1}{B}\left[\frac{\omega_{r}^{2}}{\left(\omega_{r}-k_{z} u_{0 p}\right)^{2}}\left(\frac{v_{p}}{\left(\omega_{r}-k_{z} u_{0 p}\right)}+\sqrt{\frac{\pi}{2}}\left(\frac{\omega_{r}-k_{z} u_{0 p}}{k_{z} v_{T p}}\right)^{3} \mathrm{e}^{-\zeta_{p, r}^{2}}\right)+D\right]
\end{aligned}
$$

In the above

$$
\begin{aligned}
& A=\frac{\omega_{r}^{2}}{\left(\omega_{r}-k_{z} u_{0 p}\right)^{2}}+\sum_{s} \alpha_{s}\left(\frac{u_{\perp s}}{v_{t s}}\right)^{3} \frac{\cos \left(\frac{3}{2} \theta\right)}{\left[1+4\left(u_{\perp s} / v_{t s}\right)^{2}\right]^{3 / 4}} \\
& B=\frac{\omega_{r}^{3}}{\left(\omega_{r}-k_{z} u_{0 p}\right)^{3}}-\sum_{s} \frac{\alpha_{s}}{2}\left(\frac{u_{\perp s}}{v_{t s}}\right)^{3} \frac{\cos \left(\frac{3}{2} \theta\right)}{\left[1+4\left(u_{\perp s} / v_{t s}\right)^{2}\right]^{3 / 4}}
\end{aligned}
$$

and

$$
D=\sum_{s} \alpha_{s}\left(\frac{u_{\perp s}}{v_{t s}}\right)^{3} \frac{\sin \left(\frac{3}{2} \theta\right)}{\left[1+4\left(u_{\perp s} / v_{t s}\right)^{2}\right]^{3 / 4}}
$$

with

$$
\alpha_{s}=\frac{m_{p}}{m_{s}} \frac{n_{s}^{r}}{n_{p}}
$$

and

$$
\theta=\tan ^{-1}\left(\frac{2 u_{\perp s}}{v_{t s}}\right)
$$

In (10d), $n$ denote the densities and $m$, the masses of the constituents.

\subsection{Small Thermal Spreads $\left(v_{t s}<u_{\perp s}\right)$}

In this sub-section, we derive expressions for the real frequency and the growth/damping rate of the IA wave when the ring ions have low thermal spreads. The contributions from the solar wind electrons and protons remain unchanged; the contributions of the ring ions can be derived keeping in mind the above limit of $v_{t s}<u_{\perp s}$.

Corresponding to (8) and (9), the relevant expressions, for the real frequency and the growth/damping rate are now,

$$
\omega_{r}^{2}=\omega_{p p}^{2} \frac{k^{2} \lambda_{D e}^{2}}{\left(1+k^{2} \lambda_{D e}^{2}\right)} A^{\prime}
$$

and

$$
\begin{aligned}
\gamma= & -\sqrt{\frac{\pi}{8}} \frac{\omega_{r}}{\left(1+k^{2} \lambda_{D e}^{2}\right)}\left[\frac{\omega_{r}-k_{z} u_{0 e}}{k_{z} v_{T e}} \Gamma_{0}\left(b_{e}\right) \mathrm{e}^{-\zeta_{e, r}^{2}} \times\left(1+\sqrt{\frac{\pi}{2}} \frac{v_{e}}{k_{z} v_{T e}} \Gamma_{0}\left(b_{e}\right) \mathrm{e}^{-\zeta_{e, r}^{2}}\right) \frac{A^{\prime}}{B^{\prime}}\right] \\
& -\frac{\omega_{r}}{2} \frac{1}{B^{\prime}}\left[\frac{\omega_{r}^{2}}{\left(\omega_{r}-k_{z} u_{0 p}\right)^{2}}\left(\frac{v_{p}}{\left(\omega_{r}-k_{z} u_{0 p}\right)}+\sqrt{\frac{\pi}{2}}\left(\frac{\omega_{r}-k_{z} u_{0 p}}{k_{z} v_{T p}}\right)^{3} \mathrm{e}^{-\zeta_{p, r}^{2}}\right)+D^{\prime}\right]
\end{aligned}
$$


In (11) and (12)

$$
\begin{aligned}
& A^{\prime}=\frac{\omega_{r}^{2}}{\left(\omega_{r}-k_{z} u_{0 p}\right)^{2}}-\sum_{s} \frac{\alpha_{s}}{4}\left(\frac{u_{\perp s}}{v_{t s}}\right)^{3 / 2} \\
& B^{\prime}=\frac{\omega_{r}^{3}}{\left(\omega_{r}-k_{z} u_{0 p}\right)^{3}}+\sum_{s} \frac{\alpha_{s}}{8}\left(\frac{u_{\perp s}}{v_{t s}}\right)^{3 / 2}
\end{aligned}
$$

and

$$
D^{\prime}=\sum_{s} \frac{\alpha_{s}}{4}\left(\frac{u_{\perp s}}{v_{t s}}\right)^{3 / 2}
$$

In deriving (8) and (11), the only other approximation used is $\omega_{r} \approx k_{\perp} u_{\perp s} \quad$ [14].

\section{Formula for Group Velocity}

In this section we derive an expression for the group velocity of propagation of the IA wave.

From (10a) and (13a) we find that the contribution of the protons is identical; the contributions of the ring current ions, though different, are independent of the wave vector $\boldsymbol{k}$. Hence, fortuitously, the expression for group velocity is independent of the state of thermalization of the ring ions. Thus differentiating (8) and (11) we find the expression for the group velocity to be identical for the limiting cases. The final simplified expression for the group velocity of propagation of the IA wave is

$$
v_{g r}=\frac{\omega_{r}}{k}\left[1-\frac{1}{\beta} \frac{k^{2} \lambda_{D e}^{2}}{\left(1+k^{2} \lambda_{D e}^{2}\right)}\right]
$$

where

$$
\beta=1+\omega_{p p}^{2} \frac{k^{2} \lambda_{D e}^{2}}{\left(1+k^{2} \lambda_{D e}^{2}\right)} \frac{k_{z} u_{0 p}}{\left(\omega_{r}-k_{z} u_{0 p}\right)^{3}}
$$

As a check on (15) we note that when $u_{0 p}=0$ (non-drifting protons) $\beta=1$ and the group velocity is less than the phase velocity for a finite $|k|$. On the other hand, for large $|k|, v_{g r}=0$ and the IA wave turns into IA oscillations.

\section{Discussion}

Our dispersion relations (8) and (11) and the expressions for the growth/damping rate namely (9) and (12) are very general expressions. Neglect of the contributions of the ring current ions and the higher order terms, reduce them to the standard expressions, for real frequency and the growth/damping rate [23]. Also when these terms are neglected, the IA wave is driven unstable by the electron drift velocity which is well known [3].

\section{Results}

We plot the growth/damping rate and the real frequency for typical parameters observed at comet Halley. They are $n_{p}=4.95 \mathrm{~cm}^{-3}$ while the ring ion densities are: $n_{\mathrm{H}^{+}}^{r}=0.5 \mathrm{~cm}^{-3}, n_{\mathrm{O}^{+}}^{r}=0.25 \mathrm{~cm}^{-3}$ and $n_{\mathrm{O}^{-}}^{r}=0.05 \mathrm{~cm}^{-3}$ [9] [10]. The temperatures are: $T_{p}=8 \times 10^{4} \mathrm{~K}$ and $T_{e}=2 \times 10^{5} \mathrm{~K}$, with a background magnetic field $B_{0}=75 \times 10^{-5} \mathrm{G}$ [9] [25]. The neutral density $n_{n}=10^{8} \mathrm{~cm}^{-3}$; the ion-neutral and electron-neutral collision frequencies were calculated using the formula $v_{e n}=n_{n} \sigma v_{T e}$ and $v_{p n}=n_{n} \sigma v_{T p}$ where $\sigma=10^{-15} \mathrm{~cm}^{2}$ is the collision cross section [23] [26]. Also $\Gamma_{0}\left(b_{e}\right)$ was set $=1$ for simplicity.

Figure 1(a) is a plot of the growth/damping rate $\left(\gamma / \omega_{r}\right)$ (9) versus $k \lambda_{D e}$, the parameters for the figure being the densities and temperatures given above. The other parameters relevant to the figure are:

$v_{t s}=320 \times 10^{5} \mathrm{~cm} \cdot \mathrm{s}^{-1}, u_{\perp s}=100 \times 10^{5} \mathrm{~cm} \cdot \mathrm{s}^{-1} \quad\left(v_{t s}>u_{\perp s}\right), u_{0 e}=1600 \times 10^{5} \mathrm{~cm} \cdot \mathrm{s}^{-1}$ and $u_{0 p}=1 \times 10^{3} \mathrm{~cm} \cdot \mathrm{s}^{-1}$. We find that growth rate of the IA wave decreases only slightly due to collisions (continuous line) in spite of the 
extremely high densities of the neutral atoms/molecules. The extremely low damping due to collisions could be a reason why IA waves are readily observed in the environments of comets. On the other hand in Figure 1(b), which is a plot of (12), $v_{t s}=350 \times 10^{5} \mathrm{~cm} \cdot \mathrm{s}^{-1}, u_{\perp s}=420 \times 10^{5} \mathrm{~cm} \cdot \mathrm{s}^{-1}\left(v_{t s}<u_{\perp s}\right)$ the IA wave is damped; the damping increases with collisions(continuous line). This is as expected as one of the mechanisms for wave damping is the collisional damping of waves. The peak in the growth/damping rate also indicates a resonance between the streaming particles and the wave.

Figure 2 is a plot of the real frequency $\omega_{r}$ vs. $\boldsymbol{k}$. In both panels the solid line (curve (a)) is for a plasma containing protons and hydrogen ring ions (with densities $n_{p}=4.95 \mathrm{~cm}^{-3}$ and $n_{\mathrm{H}^{+}}^{r}=0.5 \mathrm{~cm}^{-3}$ ). Similarly, curve (b) is for the situation where the hydrogen ring is replaced by the $\mathrm{O}^{-}$ring, the densities being $n_{p}=4.95 \mathrm{~cm}^{-3}$ and $n_{\mathrm{O}^{-}}^{r}=0.25 \mathrm{~cm}^{-3}$. The other parameters are $v_{t \mathrm{~s}}=320 \times 10^{5} \mathrm{~cm} \cdot \mathrm{s}^{-1}, u_{\perp s}=100 \times 10^{5} \mathrm{~cm} \cdot \mathrm{s}^{-1}$ $\left(v_{t s}>u_{\perp s}\right)$ for the left panel (Figure 2(a), Equation (8)) while $v_{t s}=350 \times 10^{5} \mathrm{~cm} \cdot \mathrm{s}^{-1}, u_{\perp s}=420 \times 10^{5} \mathrm{~cm} \cdot \mathrm{s}^{-1}$ $\left(v_{t s}<u_{\perp s}\right)$ for the right panel (Figure 2(b), Equation (11)). We find that there is an appreciable difference in frequencies as the thermalization of the $\mathrm{O}^{-}$ring ions varies; the consequent variation in the phase and group velocities of the IA wave in the presence of the $\mathrm{O}^{-}$ring ions could give us an idea of the thermalization of the $\mathrm{O}^{-}$ions.

In Figure 3 a plot of the group velocity $v_{g r}(14)$ vs. $\boldsymbol{k}$ is given. In both panels curve (a) (continuous line) is for a plasma containing protons $\left(n_{p}=4.95 \mathrm{~cm}^{-3}\right)$ and hydrogen ring ions $\left(n_{\mathrm{H}^{+}}^{r}=0.5 \mathrm{~cm}^{-3}\right)$, while, curve (b) is for a plasma where the hydrogen ring is replaced by the $\mathrm{O}^{-}$ring $\left(n_{\mathrm{O}^{-}}^{r}=0.25 \mathrm{~cm}^{-3}\right)$. The other parameters are: for Figure 3(a), $v_{t s}=320 \times 10^{5} \mathrm{~cm} \cdot \mathrm{s}^{-1}, u_{\perp s}=100 \times 10^{5} \mathrm{~cm} \cdot \mathrm{s}^{-1}\left(v_{t s}>u_{\perp s}\right)$ and $v_{t s}=350 \times 10^{5} \mathrm{~cm} \cdot \mathrm{s}^{-1}$,

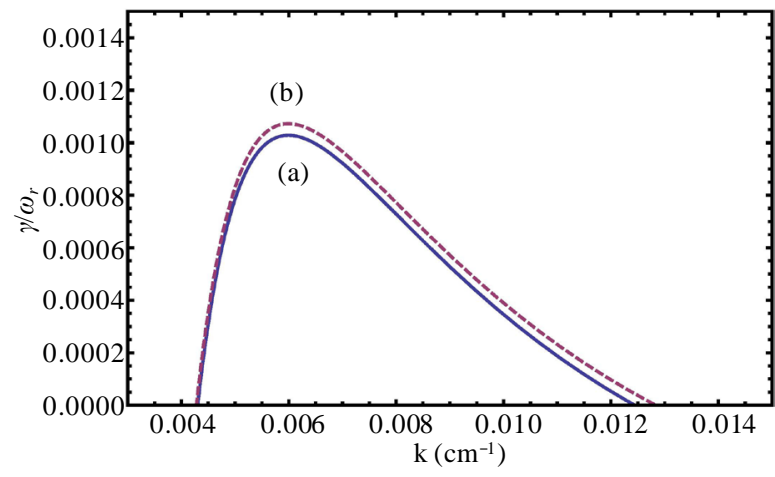

(a)

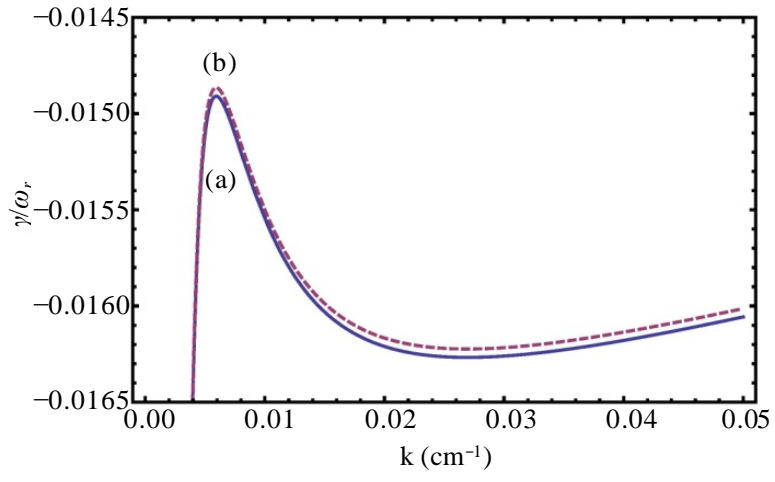

(b)

Figure 1. Plot of the growth/damping rates $\left(\gamma / \omega_{r}\right)$ versus $k \lambda_{D e}$ for (a) $v_{t s}>u_{\perp s}$ (9) and (b) $v_{t s}<u_{\perp s}$ (12). In both panels curve (a) is for the case with collisions and curve (b) for the case without collisions.

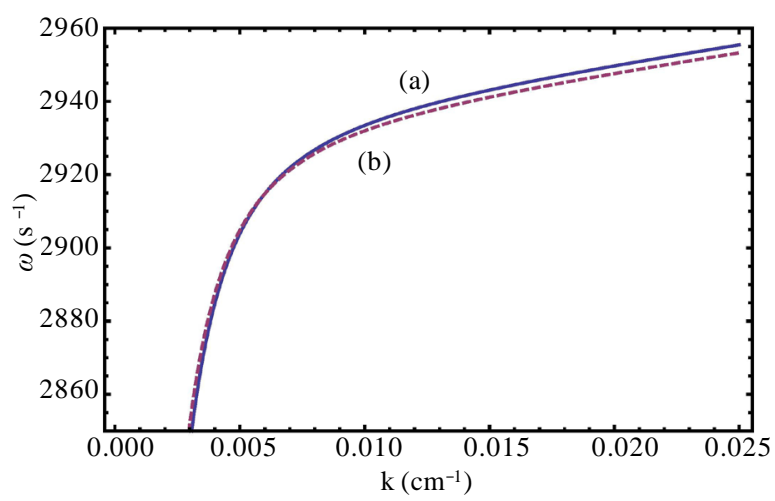

(a)

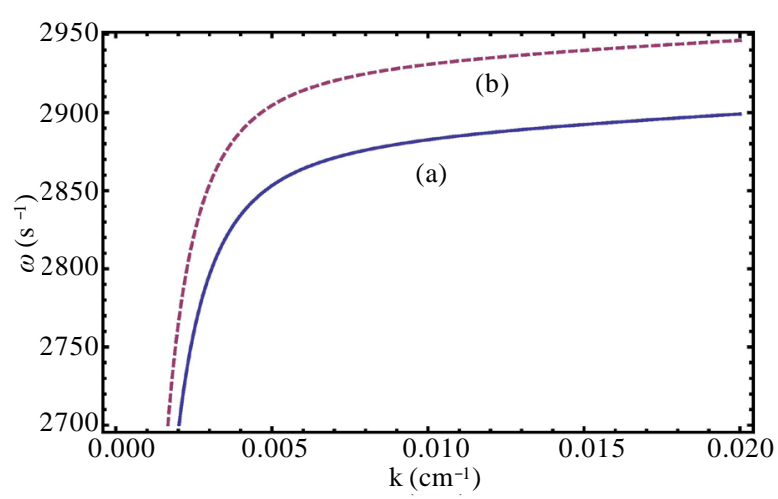

(b)

Figure 2. Plot of the real frequency $\omega_{r}$ vs. $\boldsymbol{k}$ for (a) $v_{t s}>u_{\perp s} \quad$ (8) and (b) $v_{t s}<u_{\perp s}$ (11). In both panels curve (a) is for a plasma containing protons and hydrogen ring and curve (b) for a plasma containing protons and negatively charged oxygen ring. 
$u_{\perp s}=420 \times 10^{5} \mathrm{~cm} \cdot \mathrm{s}^{-1} \quad\left(v_{t s}<u_{\perp s}\right)$ for Figure 3(b). We find that the difference in the group velocity of the IA wave, for a plasma containing a hydrogen ring or a negatively charged oxygen ring, is more profound when $v_{t s}<u_{\perp s}$ than when $v_{t s}>u_{\perp s}$; as seen in the case of real frequencies (Figure 2). The group velocity of the IA wave, in a plasma consisting of a hydrogen (or oxygen) ring, is also distinctively different for $v_{t s}>u_{\perp s}$ and $v_{t s}<u_{\perp s}$ which can be utilized to estimate the thermalization of the $\mathrm{O}^{-}$ions.

In Figure 4 the group velocity $v_{g r}$ (14) as function of (a) the ring speed $u_{\perp s}$ when $v_{t s}>u_{\perp s}$ and (b) the thermal spread $v_{t s}$ when $v_{t s}<u_{\perp s}$ is studied. In both panels curve (a) (continuous line) is for a plasma containing protons $\left(n_{p}=4.95 \mathrm{~cm}^{-3}\right)$ and hydrogen ring ions $\left(n_{\mathrm{H}^{+}}^{r}=0.5 \mathrm{~cm}^{-3}\right)$, while, curve (b) is for a plasma where the hydrogen ring is replaced by the $\mathrm{O}^{-}$ring $\left(n_{\mathrm{O}^{-}}^{r}=0.25 \mathrm{~cm}^{-3}\right)$. Here $k=0.2$ and the other relevant para-meters remain the same as in Figure 1. In panel (a) $v_{t s}=320 \times 10^{5} \mathrm{~cm} \cdot \mathrm{s}^{-1}$ and in panel (b) $u_{\perp s}=420 \times 10^{5} \mathrm{~cm} \cdot \mathrm{s}^{-1}$. The contrasting behaviour of $v_{g r}$ can be exploited to gain insight into the thermalization of the ring ions.

The variation of group velocity with the density of negatively charged oxygen ions is depicted in Figure 5. Here the parameters used are the same as in Figure 1 and $k=0.2$. Curve (a) is for the case $v_{t s}>u_{\perp s}$ and curve (b) for $v_{t s}<u_{\perp s}$. It is seen that the group velocity is insensitive to changes in the density of $\mathrm{O}^{-}$ions when the thermal spread is large; it is sensitive to the density variation when the ring speed is greater than the thermal spread.

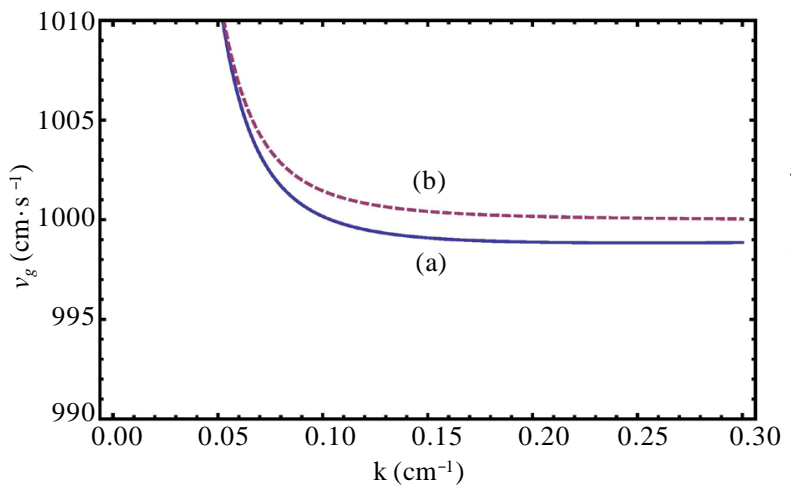

(a)

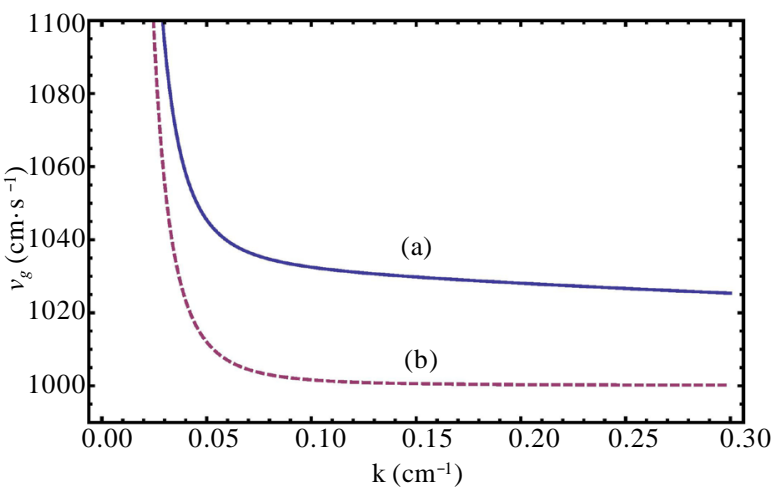

(b)

Figure 3. Plot of the group velocity $v_{g r}$ (14) vs. $\boldsymbol{k}$ for (a) $v_{t s}>u_{\perp s}$ and (b) $v_{t s}<u_{\perp s}$. In both panels curve (a) is for a plasma containing protons and hydrogen ring and curve (b) for a plasma containing protons and negatively charged oxygen ring.

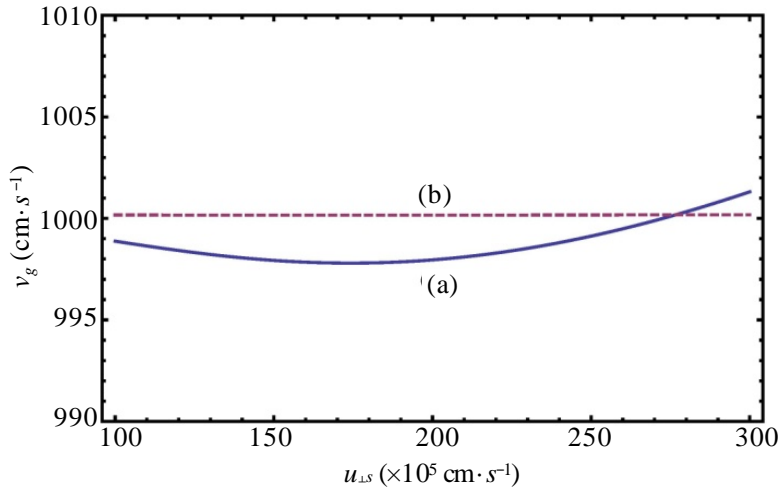

(a)

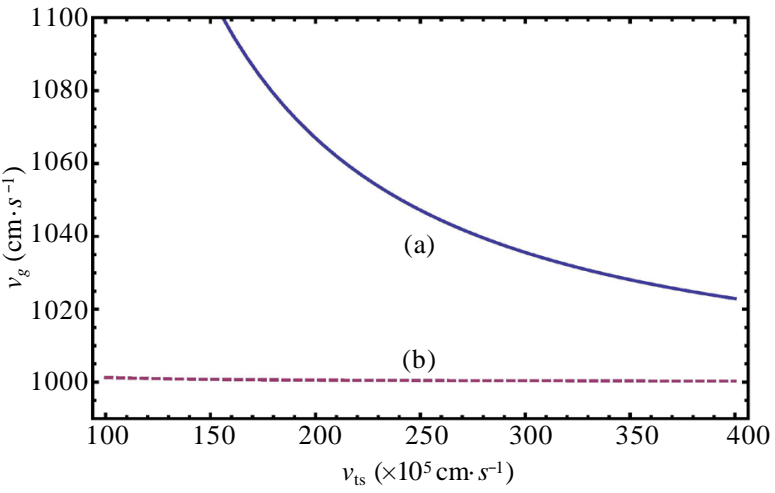

(b)

Figure 4. Plot of the group velocity $v_{g r}$ (14) as a function of (a) $u_{\perp s}\left(v_{t s}>u_{\perp s}\right)$ (b) $v_{t s}\left(v_{t s}<u_{\perp s}\right)$. In both panels curve (a) is for a plasma containing protons and hydrogen ring and curve (b) for a plasma containing protons and negatively charged oxygen ring. 


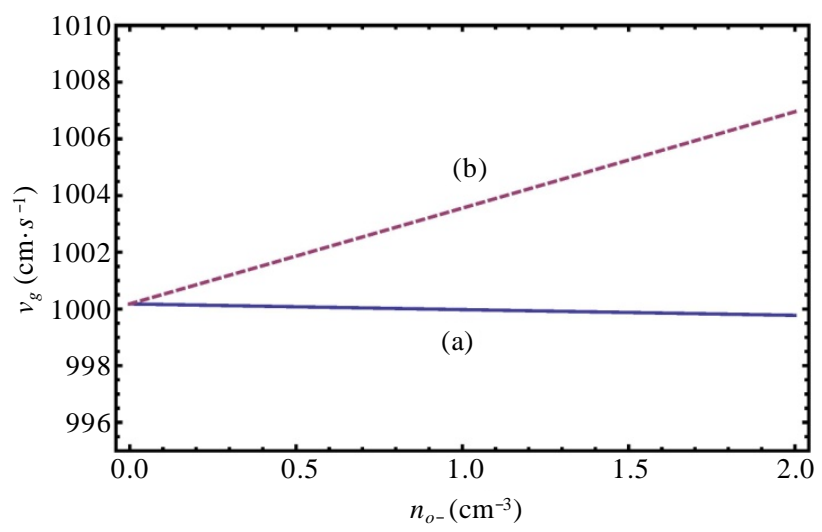

Figure 5. Plot of the group velocity $v_{g r}$ (14) as a function of the negatively charged oxygen (ring) ion density. Here curve (a) is for $v_{t s}>u_{\perp s}$ and (b) is for $v_{t s}<u_{\perp s}$.

\section{Conclusion}

We have studied the propagation characteristics of IA waves in a plasma composition that approximates very well the plasma environment of a comet. The variation of the phase as well as group velocities of the IA waves in the presence of $\mathrm{O}^{-}$ions can be used to detect their presence and also the thermalization of these ions.

\section{Acknowledgements}

We thank the referee for the very useful comments. Financial assistance from the Kerala State Council for Science, Technology \& Environment (JRF), the University Grants Commission (SAP and Emeritus Fellowship) and Department of Science and Technology (FIST and PURSE Programs) is gratefully acknowledged.

\section{References}

[1] Tonks, L. and Langmuir, I. (1929) Oscillations in Ionized Gases. Physical Review, 33, 195-210. http://dx.doi.org/10.1103/PhysRev.33.195

[2] Castro, J., McQuillen, P. and Killian, T.C. (2010) Ion Acoustic Waves in Ultracold Neutral Plasmas. Physical Review Letters, 105, Article ID: 65004. http://dx.doi.org/10.1103/PhysRevLett.105.065004

[3] Stix, T.H. (1992) Waves in Plasmas. 2nd Edition, Springer, New York,

[4] Liu, Z., Liu, L. and Du, J. (2009) A Nonextensive Approach for the Instability of Current-Driven Ion-Acoustic Waves in Space Plasmas. Physics of Plasmas, 16, Article ID: 072111. http://dx.doi.org/10.1063/1.3176516

[5] Koepke, M.E. (2002) Contributions of Q-Machine Experiments to Understanding Auroral Particle Acceleration Processes. Physics of Plasmas, 9, 2420-2427. http://dx.doi.org/10.1063/1.1456068

[6] Cranmer, S. R., van Ballegooijen, A.A. and Edgar, R.J. (2007) Self-Consistent Coronal Heating and Solar Wind Acceleration from Anisotropic Magnetohydrodynamic Turbulence. Astrophysical Journal Supplement Series, 171, 520 551. http://dx.doi.org/10.1086/518001

[7] Yamada, M. and Raether, M. (1974) Saturation of the Ion-Acoustic Instability in a Weakly Ionized Plasma. Physical Review Letters, 32, 99-102. http://dx.doi.org/10.1103/PhysRevLett.32.99

[8] Nakamura, Y., Bailung, H. and Shukla, P.K. (1999) Observation of Ion-Acoustic Shocks in a Dusty Plasma. Physical Review Letters, 83, 1602-1605. http://dx.doi.org/10.1103/PhysRevLett.83.1602

[9] Brinca, A.L. and Tsurutani, B.T. (1987) Unusual Characteristics of Electromagnetic Waves Excited by Cometary Newborn Ions with Large Perpendicular Energies. Astronomy \& Astrophysics, 187, 311-319.

[10] Chaizy, P., et al. (1991) Negative Ions in the Coma of Comet Halley. Nature, 349, 393-396. http://dx.doi.org/10.1038/349393a0

[11] Tataronis, J.A. and Crawford, F.W. (1970) Cyclotron Harmonic Wave Propagation and Instabilities: I. Perpendicular Propagation. Journal of Plasma Physics, 4, 231-248. http://dx.doi.org/10.1017/S0022377800004979

[12] Sprangle, P., Vomvoridis, J.L. and Manheimer, W.M. (1981) A Classical Electron Cyclotron Quasioptical Maser. Ap- 
plied Physics Letters, 38, 310-313. http://dx.doi.org/10.1063/1.92369

[13] Ashour-Abdalla, M. and Kennel, C.F. (1978) Nonconvective and Convective Electron Cyclotron Harmonic Instabilities. Journal of Geophysical Research, 83, 1531-1543. http://dx.doi.org/10.1029/JA083iA04p01531

[14] Akimoto, K., Papadopoulos, K. and Winske, D. (1985) Ion-Acoustic Instabilities Driven by an Ion Velocity Ring. Journal of Plasma Physics, 34, 467-479. http://dx.doi.org/10.1017/S0022377800003019

[15] Byers, J.A. and Grewal, M. (1970) Perpendicularly Propagating Plasma Cyclotron Instabilities Simulated with a One-Dimensional Computer Model. Physics of Fluids, 13, 1819-1830. http://dx.doi.org/10.1063/1.1693160

[16] Lee, J.K. and Birdsall, C.K. (1979) Velocity Space Ring-Plasma Instability, Magnetized, Part I: Theory. Physics of Fluids, 22, 1306-1314. http://dx.doi.org/10.1063/1.862733

[17] Seiler, S., Yamada, M. and Ikezi, H. (1976) Lower Hybrid Instability Driven by a Spiraling Ion Beam. Physical Review Letters, 37, 700-703. http://dx.doi.org/10.1103/PhysRevLett.37.700

[18] Mynick, H.E., Gerver, M.J. and Birdsall, C.K. (1977) Stability Regions and Growth Rates for a Two-Ion Component Plasma, Unmagnetized. Physics of Fluids, 20, 606-612. http://dx.doi.org/10.1063/1.861919

[19] Cattell, C. and Hudson, M. (1982) Flute Mode Waves near $\omega_{L H}$ Excited by Ion Rings in Velocity Space. Geophysical Research Letters, 9, 1167-1170. http://dx.doi.org/10.1029/GL009i010p01167

[20] Akimoto, K., Papadopoulos, K. and Winske, D. (1985) Lower-Hybrid Instabilities Driven by an Ion Velocity Ring. Journal of Plasma Physics, 34, 445-465. http://dx.doi.org/10.1017/S0022377800003007

[21] Scarf, F.L., Coroniti, F.V., Kennel, C.F., Gurnett, D.A., Ip, W.H. and Smith, E.J. (1986) Plasma Wave Observations at Comet Giacobini-Zinner. Science, 232, 377-381. http://dx.doi.org/10.1126/science.232.4748.377

[22] Scarf, F. (1989) Plasma Wave Observations at Comets Giacobini-Zinner and Halley. In: Tsurutani, B.T. and Oya, H., Eds., Plasma Waves and Instabilities at Comets and in Magnetospheres, American Geophysical Union, Washington D.C., 31-40.

[23] Rosenberg, M. and Merlino, R. (2007) Ion-Acoustic Instability in a Dusty Negative Ion Plasma. Planetary and Space Science, 55, 1464-1469. http://dx.doi.org/10.1016/j.pss.2007.04.012

[24] Fried, B.D. and Conte, S.D. (1961) The Plasma Dispersion Function. Academic Press, New York.

[25] Reidler, W., Schwingenschuh, K., Yeroshenko, Y.G., Styashkin, V.A. and Russell, C.T. (1986) Magnetic Field Observations in Comet Halley's Coma. Nature, 321, 288-289. http://dx.doi.org/10.1038/321288a0

[26] Curtis, C.C., Fan, C.Y., Hsieh, K.C., Hunten, D.M., Ip, W.H., Keppler, E., et al. (1987) Comet P/Halley Neutral Gas Density Profile along the Vega-1 Trajectory Measured by the Neutral Gas Experiment. Astronomy and Astrophysics, 187, 360-362. 